\title{
La web 2.0 como herramienta interactiva para mejorar la comprensión lectora de textos en inglés para estudiantes universitarios.
}

\begin{abstract}
Autores
Zoila Victoria Herrera Andrade ${ }^{1}$, Esthela Isabel Colcha Guashpa ${ }^{2}$ Rocío de los Ángeles Barragán Murillo ${ }^{3}$
\end{abstract}

\section{Resumen}

Esta investigación tiene como finalidad mejorar la comprensión lectora de los estudiantes de tercer nivel de la Escuela de Ingeniería Industrial de la Escuela Superior Politécnica de Chimborazo, mediante el uso de la Web 2.0 como herramientas interactivas para el aprendizaje los estudiantes cuya necesidad es entender textos técnicos en ingles debido a su profesión y las demandas del mundo actual. En base a la investigación previa se evidencia que el nivel lector de los estudiantes no es óptimo. La realidad existente en este grupo refleja la falta de capacitación metodológica y poco uso de técnicas interactivas para la enseñanza aprendizaje por parte de los docentes. Esta investigación pretende que los docentes hagan uso de estas técnicas con sus estudiantes para obtener resultados positivos en la comprensión lectora con el objetivo de lograr un aprendizaje significativo a través de actividades en las que el estudiante está involucrado en todo momento. Existe una fuerte correlación entre la lectura y un buen desempeño académico. En otras palabras, un estudiante que lee es muy probable que tenga éxito escolar en comparación con un estudiante que no tiene hábitos de lectura.

Palabras Claves: Web 2.0- Herramientas interactivas-Idioma Inglés-Comprensión lectoraTextos técnicos.

\begin{abstract}
The aim of this work is to improve the reading comprehension of the third level students of the Industrial Engineering School of Escuela Superior Politécnica de Chimborazo, through the use of Web 2.0 as interactive tools for learning students whose need is to understand technical texts in English due to their profession and the demands of today's world. Based on previous research it is evident that the reading level of the students is not optimal. The existing reality in this

\footnotetext{
${ }^{1}$ Escuela Superior Politécnica de Chimborazo, Chimborazo, Ecuador, zoila.herrera@espoch.edu.ec

${ }^{2}$ Escuela Superior Politécnica de Chimborazo, Chimborazo, Ecuador, e_colcha@espoch.edu.ec

${ }^{3}$ Escuela Superior Politécnica de Chimborazo, Chimborazo, Ecuador, robarragan@espoch.edu.ec
} 
group reflects the lack of methodological training and little use of interactive techniques for the teaching-learning process by teachers. This research aims for teachers to use these techniques with their students to obtain positive results in reading comprehension with the aim of achieving meaningful learning through activities in which the student is involved at all times. There is a strong correlation between reading and good academic performance. In other words, a student who reads is very likely to have school success compared to a student who has no reading habits.

Palabras Claves: Web 2.0- interactive tools-English language-Reading comprehensiontechnical texts.

\section{Introducción}

El idioma inglés y las nuevas tecnologías están íntimamente ligados debido a que los tiempos han cambiado y con ello la forma en la que los estudiantes aprenden. En tal virtud es necesario que los docentes aprovechen y apliquen herramientas tecnológicas como soporte para el aprendizaje mediante el uso de las nuevas tecnologías como técnicas pedagógicas interactivas para mejorar la comprensión lectora de textos técnicos en inglés a través del uso de una variedad de servicios y aplicaciones que ofrece web 2.0 para el aprendizaje.

El rol que tiene el docente tiene gran importancia en el desarrollo de sus estudiantes, pues este debe estar constantemente actualizando sus conocimientos en cuanto a pedagogía y tecnología por cuanto el mundo es más dinámico, va cambiando y la forma de enseñar no es la misma que la del pasado, razón por la cual es muy necesario que los docentes vayan a la par con las nuevas tecnologías, permitiendo contar con técnicas pedagógicas que contribuyan a la formación de los estudiantes.

Los estudiantes deben estar en capacidad de leer textos en inglés en su vida profesional, sin embargo, en base al estudio realizado en el grupo de estudiantes de la ESPOCH, se revela que el nivel lector es deficiente, ante tales circunstancias en esta investigación se presenta un documento que contiene técnicas interactivas basadas en los servicios y aplicaciones que brinda la web 2.0 con el fin de mejorar los hábitos de lectura, así como también motivar al estudiante a leer.

Al momento no se ha desarrollado ningún trabajo de investigación sobre técnicas interactivas encaminado a mejorar la comprensión lectora a nivel nacional. Existe una investigación de la Universidad de Chile sobre el Uso de las Tics como apoyo Pedagógico en el Proceso de Enseñanza-Aprendizaje de la Lecto-Escritura.

La guía didáctica va encaminada a facilitar el proceso de enseñanza-aprendizaje de los docentes de inglés que trabajan con estudiantes puesto que a través de la utilización de las técnicas que en ella se describen aparte de desarrollar la lectura también motiva al alumno a trabajar en una comunidad digital en colaboración de maestros y compañeros. 


\section{La Web 2.0}

Este término apareció en 2004. Tim O’Reilly nació en Irlanda, pero creció en San Francisco, Estados Unidos, fue el fundador de la editorial de libros de informática O'Reilly Media, es conocido por haber acuñado el término Web 2.0. Surge en contraste de los navegadores ofreciendo posibilidades diferentes a Altavista, correo de Hotmail, encarta o Netscape navegador 4.7. El contenido de la web 2.0 es el resultado de un trabajo colectivo contribuyendo al pensamiento crítico, autonomía, iniciativa, trabajo colaborativo y responsabilidad individual. (Gónzalez Vallés, 2015)

Con el surgimiento de la tecnología y la información, se ha desarrollado nuevas formas de aprender y enseñar. Tanto los roles de los maestros como de los estudiantes han cambiado debido a la necesidad de pasar de la recepción de conocimientos a la creación de proyectos múltiples de aprendizajes.

Batista (2007) señala que en el modelo pedagógico se cambia de la habilidad también cultivada, de la escucha para las notas de cuaderno bien compiladas y resumidas hacia las mega habilidades comunicativas (leer, entender, escribir y circular mensajes) por medio electrónico. (p. 31). A raíz de esto se formula un nuevo modelo pedagógico para el aprendizaje electrónico en la sociedad del conocimiento denominado modelo pedagógico ciber náutico.

Cuando se hace uso de entornos virtuales para el aprendizaje, el autor Portilla (2011), manifiesta: Al estudiar en un ambiente virtual, se deberá conformar un medio apoyado por las TIC. Este ambiente de aprendizaje debe ser dinámico, flexible, colaborativo, abierto, con contenidos formativos y acceso a base de datos.

Un aspecto relevante del aprendizaje colaborativo es la interacción entre los participantes, cada uno aporta con criterios, opiniones, etc., que ayudan en la restructuración cognitiva. Permite el intercambio de ideas, resolución de problemas y el logro de objetivos individuales y grupales. También se señala que una vez que el estudiante alcanza una meta con la ayuda de un compañero, luego logrará hacerlo por sí solo. (Atuesta, 2007)

\section{Internet}

El autor Crystal (2006) manifiesta que "The Internet is an association of computer networks with common standards which enable messages to be sent from any registered computer (or host) on one network to any host on any other." (p. 3)

Internet es una gran red de computadoras conectadas entre sí por medio de líneas telefónicas, fibras ópticas, satélites o cualquier otro medio de telecomunicaciones. Es una "red de redes", el mayor grupo de computadoras interconectadas por todas las zonas del mundo que pone al usuario en contacto con miles de fuentes de información. (Moirano, 2005) 
Se desarrolló en la década de los sesenta en Estados Unidos como una red experimental que rápidamente incluyó a las fuerzas armadas, universidades, comercio y usuarios. Ahora es la red de computadoras más grande del mundo, permite que número sin precedentes de personas puedan estar comunicadas a través de correos (email), chats y páginas digitales sobre cualquier tema. Además, se puede realizar compras electrónicas, publicar anuncios, ver películas, programas de TV y cualquier tipo de entretenimiento en línea.

Con la Web 2.0 se produce un cambio en la concepción de internet dejando la representativa unidireccionalidad de sus servicios para pasar a facilitar la máxima interacción de sus usuarios y desarrollar redes sociales en las que el usuario puede expresarse, buscar y recibir información, colaborar y crear conocimiento y compartir contenidos (Graells, 2005). En efecto, el Internet ha dejado atrás la tradicional información de un solo sentido por comunicación de dos vías, representado por tres Cs: comunicación, colaboración y creatividad.

\section{Conectividad}

Con la aparición del Internet ha dado paso a que la conectividad sea posible en cualquier parte del mundo, utilizando para ello varios dispositivos estáticos y portátiles como las laptops que se convirtieron en un artefacto que se encuentra en la mayoría de los hogares, escuelas y oficinas.

Se puede decir que al hablar de técnicas interactivas hablamos nos valemos del uso de las herramientas Web 2.0 convirtiéndose en un recurso innovador y efectivo en la lectura, vocabulario, fluidez y comprensión de textos, mismos que pueden ser mejorados a través del uso de ambientes colaborativos. (Banister, 2008)

Minguell (2002) manifiesta que la interactividad debería ser:

“...una característica intrínseca de los materiales multimedia (accesibles, o no, a través de la red) que incrementase, cualitativa y cuantitativamente, la capacidad de los usuarios de intervenir en el desarrollo de las posibilidades que ofrecen los programas de manera que se pudiesen mejorar sus posibilidades de trabajo y aprendizaje.” (p. 26)

\section{Herramientas de la Web 2.0 como métodos para el aprendizaje}

Según RAE, las herramientas son un conjunto de instrumentos. Estos pueden ser utilizados para desempeñar un determinado oficio o trabajo.

Para Valerio (2001) en referencia a las herramientas manifiesta:

"Las herramientas tecnológicas, como cualquier otra herramienta, están diseñadas para facilitar el trabajo y permitir que los recursos sean aplicados eficientemente 
intercambiando información y conocimiento dentro y fuera de las organizaciones". (p. 1)

Durante los últimos años la tecnología se ha apoderado de todos los quehaceres del día a día. A los videojuegos, software, redes sociales, celulares, etc., se los puede denominar como herramientas tecnológicas y han revolucionado el modo en la que las personas viven. Aportando dentro de la educación una mejora en la calidad de formación del estudiante.

Una herramienta pedagógica es cualquier medio o elemento que interviene en el proceso de enseñanza aprendizaje de los estudiantes. Tienen como fin facilitar y optimizar la calidad de formación. Se las concibe como facilitadores. (Gutiérrez Valderrama, 2011)

Redes Sociales: Se puede denominar a las redes sociales como un espacio creado para el intercambio de información y generación de relaciones.

Según Pérez y otros (2009) dicen que "Las redes sociales constituyen un fenómeno clave para entender la dinámica de la sociedad red en la que vivimos".

Entre las redes sociales más usadas se encuentran Facebook y Twitter. También existen redes sociales para profesionales como LinkedIn, Academia, Epernicus, Lalisio, ResearchGate, Methodspace, Sciencestage, etc.

Las redes sociales permiten interactuar con otros usuarios a los que se puede o no conocer en la realidad, dinámica que se la puede hacer a través de chats, foros, spaces, etc.; convirtiéndose en un gran atractivo para los que las usan por su aspecto personal y de relación. Mientras mayor el número de usuarios miembros de la red mayor es el atractivo para los alumnos quienes desean estar en contacto permanente con sus compañeros de clases, maestros, amigos, etc. Esto permite generar un ambiente de trabajo favorable.

Blogs: Sirve para complementar el trabajo del aula. Permite otras formas de producir y publicar contenidos sin la necesidad de ser un experto en informática. Es de fácil acceso, práctico mantenimiento, es un espacio multimedia. Todo esto hace que se convierta en una puerta al descubrimiento e interacción con los lectores.

El autor Carruci (2013) expresa sobre el uso de Blogs para la comunicación y dice: "Es un recurso excelente para explorar nuevas formas de interacción con nuestros alumnos más allá de las fronteras del aula" según (Carucci, 2015) tanto docentes como estudiantes pueden hacer uso de Blogs.

El uso de los blogs depende de la creatividad de los docentes y de los objetivos que se planteen en cuanto al aprendizaje. 
Mensajería instantánea: Las tecnologías 2.0 cuentan con mensajería instantánea, ofrecen a empleados de grandes empresas la posibilidad de comunicarse instantáneamente con colegas. (Deans, 2009)

La mensajería instantánea es una técnica onmipresente, se encuentra en escuelas, colegios, universidades, centros comerciales y cines. Apareció a mediados de 1990 con el popular CQ "I seek you", seguido del Instant Messenger de AOL a finales de 1990. Hoy en día, existe una gran variedad de recursos abiertos de mensajes instantáneos o de mensajes de texto. Estas aplicaciones se pueden usar en computadoras de escritorio, teléfonos móviles y cualquier otro dispositivo inalámbrico.

Foros: Los foros pueden ser incluidos dentro de los PLE o de una plataforma de aprendizaje.

Arango (2011) manifiesta sobre los foros lo siguiente:

"Un foro es un escenario de comunicación por internet, donde se propicia el debate, la concertación y el consenso de ideas. Es una herramienta que permite a un usuario publicar su mensaje en cualquier momento, quedando visible para que otros usuarios que entren más tarde, puedan leerlo y contestarlo. A este estilo de comunicación se le llama asincrónica dada sus características de no simultaneidad en el tiempo".

Videoconferencias: En las videoconferencias, es posible compartir pantalla, usar presentaciones PowerPoint fortaleciendo así la interacción. El uso de este tipo de herramientas requiere de la motivación de los estudiantes junto con el entusiasmo del docente y el apoyo administrativo.

Chacón (2003) dice que una videoconferencia es un servicio multimedia que permite la interacción entre distintas personas o grupos de trabajo. Básicamente consiste, en interconectar mediante sesiones interactivas a un número variable de interlocutores, de forma que todos pueden verse y hablar entre sí”. (p. 2)

\section{Por contenido}

Wiki: Es un sitio web que incluye páginas que se pueden editar en línea por diferentes usuarios. Un ejemplo muy conocido es Wikipedia, es una enciclopedia que incluye artículos que han sido redactados por diferentes personas en todo el mundo. Se trabaja con contenido hipertextual, permite que el estudiante trabaje en un espacio flexible, creativo y abierto que permite la construcción del conocimiento. (Caccuri, 2013)

Las wikis son herramientas flexibles, simples y potentes en colaboración. Es un sitio web cuyas páginas pueden ser editadas por varias personas rápidamente y fácilmente desde cualquier lugar con acceso a internet. Los participantes de una wiki pueden crear, modificar o borrar un texto compartido. Por los grandes beneficios que aportan las wikis posibilitan que 
grupos de estudiantes, docentes o ambos elaboren glosario de diferentes asignaturas, reúnan contenidos, construyan colaborativamente trabajos escritos, creen sus propios libros de textos y desarrollen repositorio de recursos, etc.

Google Docs: A través de esta herramienta se comparte cualquier tipo de información y los miembros participantes para trabajar en Google Drive pueden realizar trabajo colaborativo realizando ediciones e insertando información.

El autor Caccuri (2013) se refiere a Google Dos como una herramienta que ofrece google a través de la creación de una cuenta de correo electrónico en Gmail. Incluye un procesador de textos, una hoja de cálculo, un programa para crear presentaciones con diapositivas, un editor de dibujo y una herramienta para generar formularios.

YouTube: Desde su lanzamiento en el año 2005, YouTube se ha convertido en uno de los fenómenos más impresionantes de Internet, que crece exponencialmente cada día. Según sus datos proporcionados por Google, la empresa que actualmente es propietaria del sitio, se sube a esta plataforma sesenta horas de video por minuto, se reproducen más de cuatro mil millones de videos por día. más de ochocientos millones de usuarios visitan el sitio. Un aspecto muy importante a resaltar es que es gratuito. (Caccuri, 2013)

YouTube ofrece un nuevo enfoque que provee un input linguiístico y alienta a los estudiantes a que aprendan un idioma extranjero, es una herramienta que puede ayudar a los docentes en esta tarea.

\section{Por Gestión de información}

Plataformas virtuales de aprendizaje: Los Gestores de Contenido son herramientas software utilizadas para la creación y mantenimiento de sitios web. De los gestores de contenido surgen los sistemas de gestión de aprendizaje (Learning Management System o LMS) conocidos como plataformas de aprendizaje o plataforma e-learning. Facilita la comunicación entre alumnos y profesores por medio de herramientas que permite la gestión de contenidos educativos. (Domínguez C., 2012)

Es un programa software, instalado a un servidor que sirve para administrar, distribuir, y controlar las actividades de formación presencial o e-learning. Existen algunas plataformas de aprendizaje entre ellas: Moodle, Edmodo, Sakai, Blackboard, Dokeos, ATutor, etc.

Marcadores sociales: Los marcadores sociales (social bookmarking), permiten almacenar, clasificar y compartir recursos de la red a través de listas que guardan la información, estás se clasifican y etiquetan con el etiquetado social o tags. Se puede guardar información para que el usuario pueda recordarla y compartir con otras personas. Estos marcadores pueden ser públicos o privados. (Niño, 2010) 
Delicious dió a conocer el término de marcadores sociales en 2003 y tagging (etiquetado). Yahoo Bookmark es uno de los marcadores más populares entre los usuarios junto con Delicious.

La nube: Se trata de un conjunto de herramientas y servicios a los que se puede acceder únicamente a través de internet. Estas plataformas permiten conectar diferentes dispositivos como equipos de escritorio, tablets o teléfonos celulares- y aplicaciones informáticas, para acceder a información. Se puede acceder a estos servicios desde cualquier dispositivo. Adicionalmente, toda la información queda guardada sin necesidad de contar con un dispositivo de almacenamiento. (Mohammed Abdul, 2009)

RSS: Really Simply Syndication (RSS), es un protocolo que permite que los usuarios se suscriban en línea usando un lector RSS. Este verifica las páginas web y actualiza el contenido automáticamente. Los agregadores muestran suscripciones que RSS ha agregado durante la ausencia del usuario, por tanto; el usuario no necesita visitar las páginas web una a una, puede acceder a ellas de manera fácil y rápida, constituyéndose en el medio por el cual el contenido fluye de proveedores a usuarios. Lo interesante de RSS es que él es el usuario quien determina el contenido que desea.

Se agrega un pequeño código a un sitio web, va acompañado de un icono que permite que los usuarios sepan que el contenido de la página está disponible a través de RSS. Los usuarios simplemente dan clic en el ícono u otro enlace de RSS para agregar una suscripción.

Buscadores web: El desarrollo de los navegadores fue uno de los elementos claves que contribuyeron en la evolución de la web 2.0. Un navegador es una aplicación de software que permite acceder y ver páginas web. El navegador web Mosaic, luego conocido como Netscape fue la primera interfaz gráfica de la Web. Mosaic se desarrolló para computadoras que funcionaban con el sistema operativo Unix y más tarde estuvieron disponibles para computadores personales que funcionan con sistemas operativos de Mac y Windows. Fue la primera aplicación de software que funcionaba con los dos sistemas operativos. El navegador de internet se volvió popular cuando Microsoft lo distribuyo como parte de Windows 95, el primer computador personal que incluía características que permitían el acceso a Internet. (Palomar Sánchez, 2009)

Para que los sitios web puedan ser usados por un mayor número de personas, tienen que ser visualizadas por muchos navegadores web. La competencia entre los desarrolladores web contribuyen al desarrollo continuo de los navegadores. Nuevas características se introducen en las nuevas versiones para estar a la par de la competencia. Navegadores web, como Internet Explorer 8, Safari 4, Firefox y Google Chrome han implementado características que respondan al desarrollo en las tecnologías Web, pero muchas características de la interfaz de los usuarios en estos navegadores son las mismas que se encontraban en versiones anteriores de Mosaic. (Shelly, G. B., \& Frydenberg, M., 2010) 
Equipos multimedia: Multimedia es el campo que se ocupa de integrar por medio de un ordenador el texto, gráficos, imágenes, animaciones y audio para poder ser representada, almacenada, transmitida y procesada digitalmente. Sarmiento (2007) manifiesta sobre el concepto de multimedia lo siguiente:

"El término multimedia, es igual que sucede con otros términos como medio o currículo, presenta diferentes definiciones, unas referidas al software y otras al hardware, y además contamos con otros términos...”. (p. 16)

Hardware: Grupo Educare (2011) dice que hardware es Comprender todos los dispositivos o elementos físicos (que se pueden tocar) con los cuales es construida una computadora. Incluye también los elementos mecánicos, eléctricos. Los teclados, monitores, impresoras, microprocesadores, unidades de disco, ratón, escáner y demás periféricos, son hardware.” (p. 1)

Dentro de los tipos de hardware se puede distinguir los periféricos de entrada y salida. Con respecto a los de salida tenemos: teclado, ratón, escáner, micrófono por mencionar algunos de ellos. Los de salida muestran el resultado al usuario de lo que la computadora realiza y entre ellos se encuentran. Facilitan la entrada de información al computador: monitor, impresora, etc.

Software: Grupo Educare (2011) dice sobre software que este se forma por el conjunto de instrucciones o programas. Los programas son una secuencia de órdenes que se le dan a la computadora para que haga algo. Todos los juegos de video, sistemas operativos y programas de aplicación -como procesadores de palabras o programas para internet- son software.” (p. 2)

Hipertextos: Es un conjunto de documentos que están interrelacionados a partir de un enlace o hipervínculos. Sirve para navegar de un sitio a otro. Aparece subrayado o de un color diferente. Al dar click sobre el hipervínculo se accede a otro archivo u página web.

Entre las características principales del hipertexto están la interactividad, extensibilidad, apertura. (Caccuri, 2013)

M-learning: Se refiere al uso de recursos ofrecidos por las tecnologías móviles, como celulares, smartphones, tabletas, ebook readers, notebooks, mp3. Su uso extendido se debe al aumento de uso de Internet móvil. Un gran beneficio, es que el aprendizaje puede realizar en cualquier momento y a cualquier hora, por ejemplo, cafeterías, buses, museos, etc. (Sharma \& Barrett, 2010). El celular es un recurso tecnológico que puede ser usado dentro y fuera del aula como un instrumento generador de conocimiento. Los estudiantes dedican gran cantidad de tiempo a sus celulares por lo que se lo puede utilizar como instrumento de aprendizaje.

Pizarra digital: Es una superficie sensible al tacto, la cual se encuentra conectada a un ordenador y proyecta la pantalla de este, pero a gran escala; es una pantalla táctil a gran escala. 
La mayoría de pizarras digitales están acompañadas de un software específico con rotafolios electrónicos -páginas en blanco para la creación de materiales para la enseñanza. (ITILT, 2011)

Libros digitales: Hernández y otros (2008) dicen que: "no son simplemente una versión digital de los libros impresos pues incluyen recursos multimediales interactivos y enlaces entre varios elementos del conocimiento". Los libros digitales con el tiempo no reemplazarán a los impresos, pero si los complementaran y los fortalecerán.

Marqués (2012) dice sobre los libros digitales lo siguiente:

"Proporcionan muchos nuevos recursos que mejoran la comprensión, la atención y la implicación del alumnado, facilitando una renovación metodológica orientada a la innovación didáctica...y además facilitan la adquisición de competencias TIC”. (p. 1)

Para la publicación de textos en formato de libros digitales, se puede recurrir a plataformas virtuales, blogs, sitios web personales, o ISSU, que es un espacio que permite gratuitamente la publicación de cualquier material escrito.

\section{Mind mapping}

En el caso de mapas mentales (mind mapping), se puede utilizar Mindmeister que es una herramienta de la web 2.0, que permite que los usuarios construyan mapas mentales a partir de textos. Permite la participación de varios usuarios en línea para la construcción del mapa mental. Se usa una lista ordenada de artículos. En Mindmeister se puede editar, publicar y clasificar grupalmente. (Mohammed Abdul, 2009). Edraw es otra herramienta que se puede utilizar para crear mapas mentales.

\section{Second Life}

Second Life es una cultura digital que compite cada vez más con los medios de comunicación, la vida real y la sociedad y desafía la manera en la que participamos en la creación de medios de comunicación y todas las formas de entretenimiento de los medios de comunicación. Proporciona un espacio alternativo de comunicación e interacción en un ambiente que es prácticamente un reflejo del mundo real.

Second Life es muy útil a nivel universitario puesto que permite la experimentación en situaciones que en la vida real no sería posible. Permite desarrollar comunidades, crear confianza, e incrementar el sentido de presencia en el aprendizaje. El desarrollo de destrezas en un ambiente virtual en línea es mucho más beneficioso en un ambiente real porque es un espacio diferente de los programas presenciales. (Savin-Baden, 2010) 


\section{PLE}

Un Entorno Personal de Aprendizaje (Personal Learning Environment (PLE)), es un Entorno Personal de Aprendizaje se basa en el uso de las diferentes herramientas que ofrece la web 2.0 y las redes sociales para el proceso de enseñanza aprendizaje. Permite el aprendizaje eficaz y autónomo. Martínez y Torres (2013)

Un PLE está compuesto por 3 elementos: Herramientas, mecanismos y actividades para leer, herramientas, mecanismos y actividades para hacer reflexionar haciendo y Herramientas, mecanismos y actividades para compartir y reflexionar en comunidad. La utilización de las TIC únicamente, no producen ningún aporte en la educación, es por eso que con la aparición de la web 2.0, se las utiliza ahora de una mejor manera a través de los PLE.

\section{Técnicas}

Ilhch (1972) sobre el propósito de la educación manifiesta:

"In fact, learning is the human activity which least needs manipulation by others. Most learning is not the result of instruction. It is rather the result of unhampered participation in a meaningful setting”. (p. 56)

Este autor cuestiona el hecho de si las técnicas tradicionales usadas en el proceso de enseñanza han sido efectivas.

"Una técnica es un término superordinado que se refiere a varias actividades que ya sea docentes o estudiantes realizan en el aula" (Brown, 2000). Son actividades o tareas preparadas con antelación o improvisadas por el docente sobre la base de los contenidos que se están revisando en clase. Como se puede ver, la técnica bien podría surgir de la creatividad del docente para cimentar los conocimientos de sus estudiantes.

"Se definen como formas, medios o procedimientos sistematizados y suficientemente probados, que ayudan a desarrollar una actividad, según las finalidades y objetivos pretendidos". (Junta de Andalucía). A su vez, según este documento se señala que las técnicas se usarán de acuerdo al contexto y características del grupo al que van destinadas siempre teniendo en cuenta sus necesidades, expectativas y objetivos que se espera alcanzar.

\section{Comprensión Lectora}

La comprensión lectora es la habilidad de entender un texto. Además, permite que el lector interactúe con el texto en forma significativa. A través de la comprensión lectora, se puede tener recreación y diversión. Al respecto, Thanky (2014) señala: 
"Los estudiantes deben estar en capacidad de hacer uso de lo que leen para expandir el conocimiento en materias relacionadas a su acción estudiantil como por ejemplo en este caso dinámica, materiales, etc. Deben estar en capacidad de entender y usar la información que leen en diversos propósitos. Por tal razón, los graduados de Ingeniería requieren una gama de habilidades para ir acorde al medio ambiente del Nuevo milenio". (p. 1)

Crespo (2004) manifiesta que existen tres teorías significativas de la comprensión lectora:

- La lectura como conjunto de habilidades o transferencia de información: Se trata del conocimiento de palabras. Comprende la comprensión y evaluación. La comprensión trata de entender lo implícito y evaluación de valorar la calidad del texto, las ideas e intención del autor.

- La lectura como proceso interactivo: Surge a partir de los avances de la psicolingüística y el cognitivismo a finales de los años setenta. El lector en base a sus conocimientos previos, debe interactuar con el texto y construir su significado.

- La lectura como proceso transaccional: Es la relación recíproca entre el lector y el texto. Es la confluencia temporal entre el lector y el texto. Existe un mayor significado del texto debido a que constituye la suma de los esquemas del lector y el texto.

\section{Textos técnicos}

"Los textos científicos-técnicos pertenecen a sublinguales entroncados en dominios concretos de la sociedad y por lo tanto muestran diferencias interlingüísticas y estructura entre ellos" (García, A. 2007).

Los textos técnicos son más directos que otros textos desde el punto de vista culturalpragmático, ciencia y tecnología ya que trascienden culturas y representan un punto de referencia para la humanidad. Los textos técnicos tienden a usar un registro formal en niveles variados de terminología que lo diferencia del registro standard. Cuando se escucha hablar de un texto técnico, inmediatamente se asume que es difícil de comprender.

\section{Técnicas de lectura}

Extraer la idea principal: No es necesario leer todo el párrafo. Se puede leer la primera línea de cada párrafo que es en donde se encuentra the topic sentence y así tomar nota para al final determinar la idea general del texto.

Scanning: Es la mejor estrategia para buscar información específica en un texto. Se debe mover los ojos de arriba a abajo recorriendo el texto hasta encontrar la palabra o palabras que 
se desea. No siempre es necesario leer todo el texto para encontrar determinado tipo de información.

Skimming: Se trata de localizar información específica rápidamente. Una estrategia útil es leer el texto rápidamente para obtener la idea principal. Aquí es preciso decidir qué tipo de información es importante para ser leída con más detalle dependiendo del propósito de la lectura.

Activación de esquema e interés antes de la lectura: Hacer conexión con lo que el estudiante ya conoce de modo que al momento de leer tenga sentido lo que lee.

Predicción: Es necesario pensar sobre lo que se va a leer antes de hacerlo. No es buena idea empezar a leer un texto inmediatamente. Una forma de facilitar el proceso lector es pensar acerca de las palabras que podrían aparecer en el texto. El título ayuda a enfocar las ideas.

Entender vocabulario desconocido: Es acercase lo máximo posible al significado de la palabra de acuerdo al contexto. No se recomienda el uso del diccionario.

\section{Metodología}

La presente investigación es de Tipo No experimental, con la cual se observa los hechos en su contexto natural, aplicando para ello la investigación aplicada, con la cual se va a realizar el análisis del problema educativo que afecta a los estudiantes de educación superior, para investigarlo y proponer una alternativa de solución. Es de nivel correlacional; porque se presenta en el proceso investigativo la respuesta al problema a través de la relación entre variables (causa - efecto), así también por el lugar, la investigación es de campo, al realizar el trabajo en el lugar en donde se producen los hechos, estudiantes del Tercer Nivel, de la carrera de Ingeniería Industrial de la Escuela Superior Politécnica de Chimborazo, y de origen bibliográfica documental, su propósito es profundizar los diferentes enfoques teóricos de diversos autores, basándose en documentos de fuentes primarias y secundarias.

Los métodos empleados en el proceso investigativo son el Científico, el cual se basa en los principios y elementos del método científico empleados según su estructura y procedimientos. El Analítico-Sintético, se utiliza en el desarrollo de la investigación permitiendo el procesamiento de toda la información, su análisis, interpretación de resultados y elaboración de conclusiones y recomendaciones. El método Estadístico, que sirve para el análisis de los datos, apoyado en el programa informático Excel, mediante el uso de la Estadística Descriptiva e Inferencial, con lo que se procede a comprobar la hipótesis y elaborar las conclusiones.

La población considerada para el estudio está formada por estudiantes de tercer nivel de inglés de la Escuela de Ingeniería Industrial de la Escuela Superior Politécnica de Chimborazo, misma que está compuesta por 104 estudiantes de tercer nivel de Inglés de la Escuela de Ingeniería 
Industrial, 5 docentes que imparten clases de inglés en esta escuela y 4 autoridades de la ESPOCH dando un total de 113.

Entre las Técnicas se aplicó la encuesta a los estudiantes y la entrevista a las autoridades y docentes de la institución, diseñando para ello cuestionarios como instrumentos de recolección de datos

Para la validación de la información obtenida se

Tanto para la validación como para la confiabilidad de la información se consideró el juicio de 3 expertos en el área y del manejo de instrumentos, para lo cual se les entrego la documentación correspondiente más el formulario para la validación.

\section{Resultados}

Gráfico 1: Técnicas interactivas

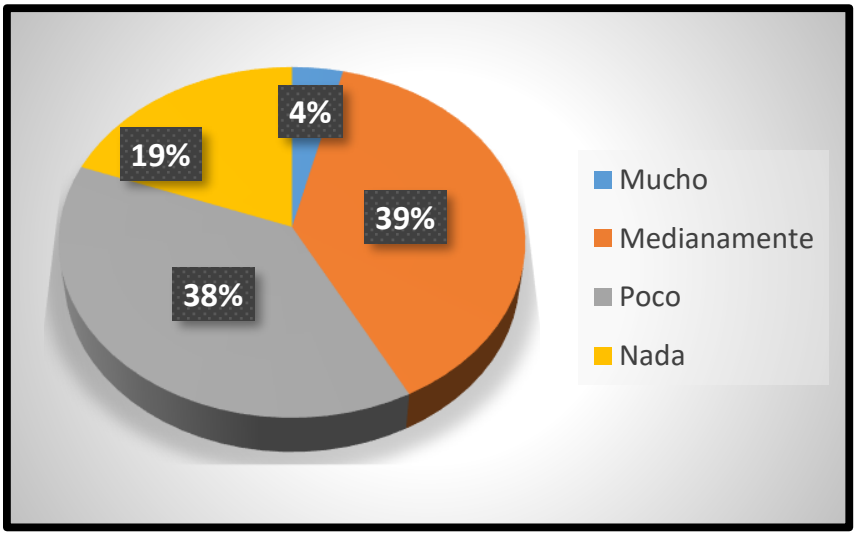

Fuente: Secretaría del Centro de Idiomas ESPOCH

Gráfico 2: Uso de técnicas interactivas

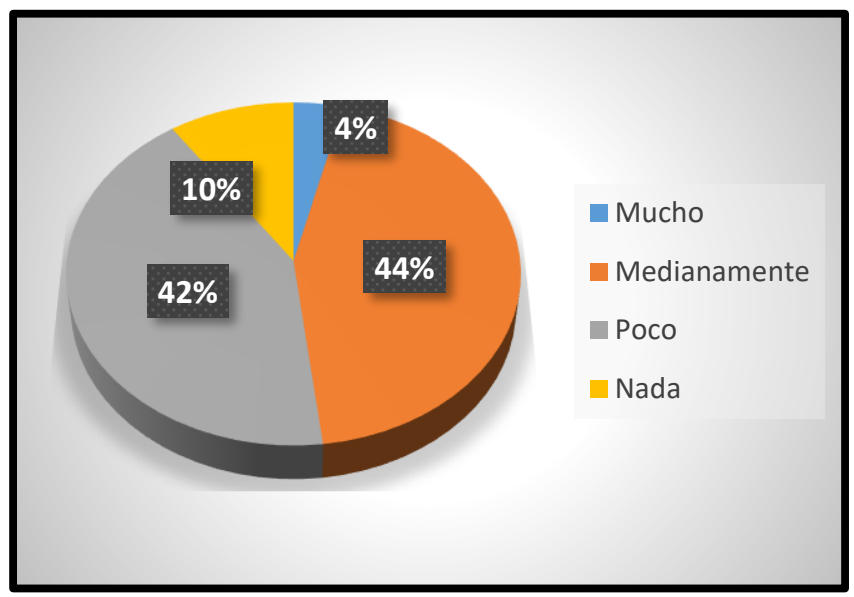

Fuente: Secretaría del Centro de Idiomas ESPOCH 
Gráfico 3 Entornos virtuales para el aprendizaje

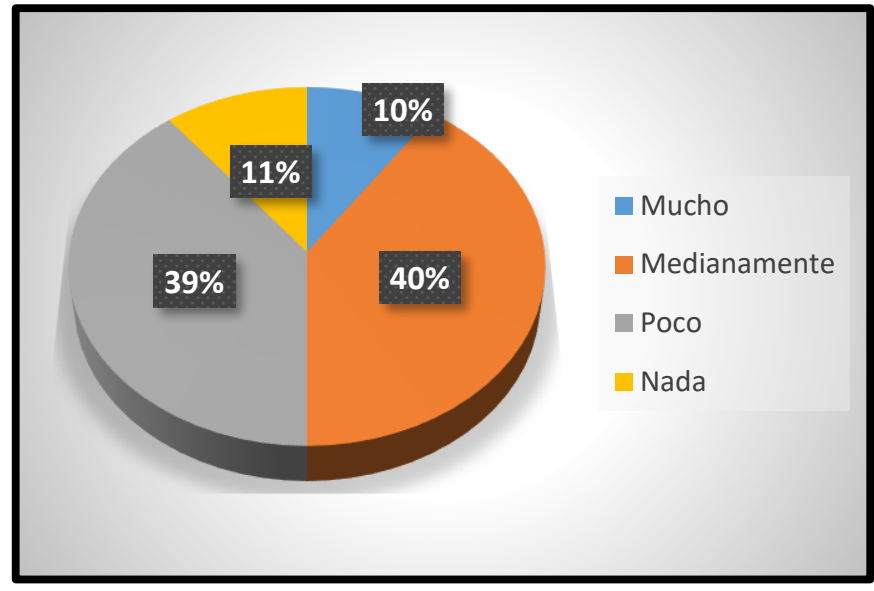

Fuente: Secretaría del Centro de Idiomas ESPOCH

Gráfico 4 Entornos virtuales para la comprensión lectora

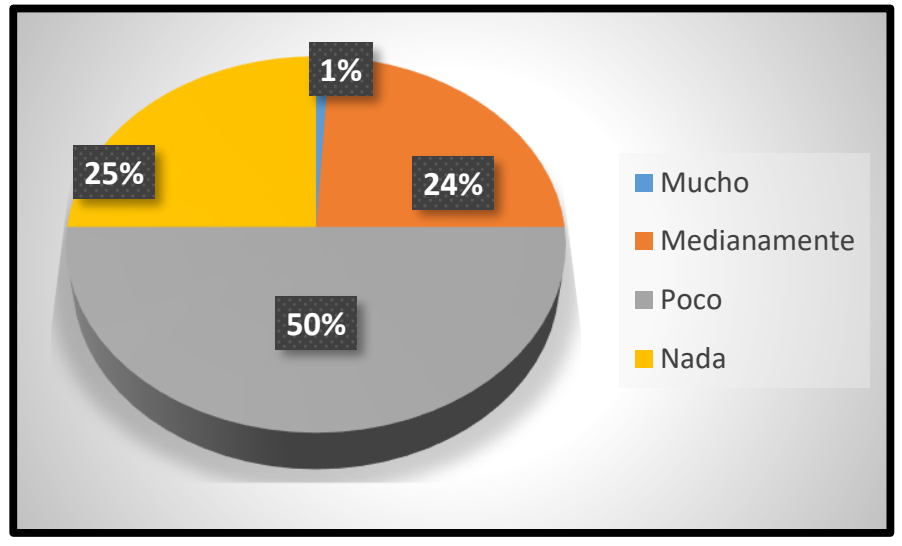

Fuente: Secretaría del Centro de Idiomas ESPOCH

Gráfico 5 Inferir significados

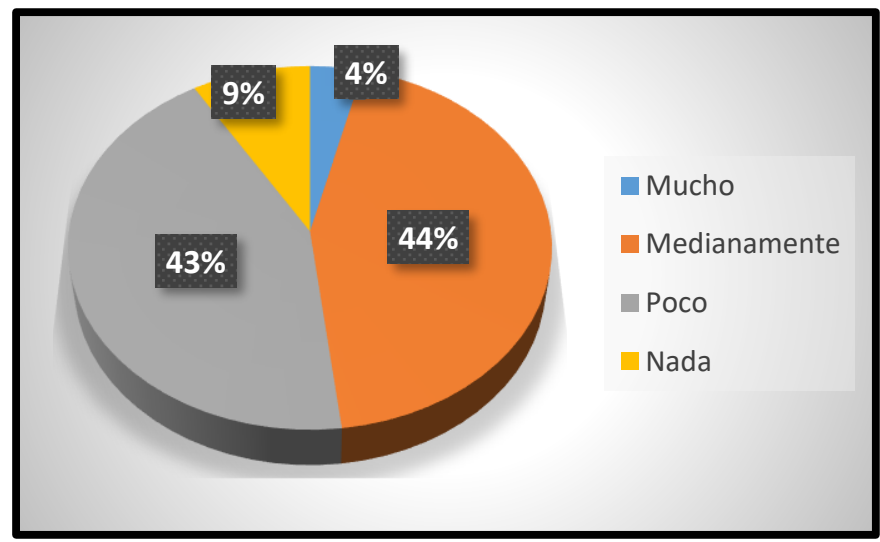

Fuente: Secretaría del Centro de Idiomas ESPOCH

\section{Validación}

Se considera el juicio de 3 docentes expertos en el área y manejo de instrumentos, como son

- MSc. Adriana Cundar 
- MSc. Fanny Zambrano

- MSc. Sandra Abarca

A quienes se les entregó la documentación correspondiente para el registro de su validación y confiabilidad de los instrumentos elaborados para su correcta aplicación.

- Oficio

- Instrucciones

- Preguntas directrices

- Objetivos

- Matriz de operacionalización de variables

- Instrumentos de Investigación

- Formulario para la validación.

\section{Conclusiones}

- Los estudiantes pese a ser jóvenes y considerados nativos digitales, así como usuarios de nuevas tecnologías, no se las usa plenamente para el aprendizaje del idioma inglés.

- Los docentes por falta de conocimientos o de recursos no usan tecnología en clases dificultando así el aprendizaje, ya que se rigen al aprendizaje con metodología tradicional para la enseñanza.

- Se puede decir que no existe un sólido conocimiento y uso de los entornos virtuales para el aprendizaje en general y por ende del idioma inglés. Es interesante evidenciar que estudiantes jóvenes no están familiarizados con ambientes virtuales.

- Los docentes de inglés no utilizan técnicas interactivas para impartir sus clases, por decirlo el uso de plataformas virtuales, por tanto; los estudiantes no conocen sobre técnicas interactivas para aprender inglés mediante el uso de herramientas que proporciona la web 2.0.

- El estudiantado en inglés debe, tener conocimiento sobre la comprensión lectora para que sea de mayor entendimiento a la lectura, sin embargo, no cuenta con jornadas de capacitación sobre técnicas de lectura a sabiendas de que esta destreza es de suma importancia ya que la bibliografía concerniente a la carrera se encuentra mayoritariamente en inglés.

- Los estudiantes en su mayoría usan diccionario para traducir palabra por palabra, lo cual hace que no puedan continuar con la dinámica del aprendizaje secuencial por tanto si no pueden inferir significados por el contexto, el estudiante al desconocer palabras podría ignorarlas u omitir información importante, dejando de lado la información aprendida después de leer un texto en otros contextos. 


\section{Referencias Bibliográficas}

Arango M., M. L. 2011. Foros virtuales como estrategia de aprendizaje. . s.1. : Retrieved from http://tic.sepdf.gob.mx/micrositio/micrositio2/archivos/ForosVirtuales.pdf, 2011.

Atuesta, C. M. 2007. Hacia una comunidad interactiva. Medellín : Editorial Universidad EAFIT, 2007.

Banister, S. 2008. Web 2.0 Tools in reading classroom: . Teacher exploring literacy in the $21 \mathrm{st}$ century. International Journay of Technology in Teaching and Learning, 109-116 : s.n., 2008.

Batista, E. 2007. Lineamientos pedagógicos para la enseñanza y el aprendizaje. Colombia : Universidad de Colombia, 2007.

Crespo Picó, Mila. 2004. El pretexto del texto. La comprensión lectora como proceso vehicular hacia otras destrezas. [En línea] 2004. http://cvc.cervantes.es/ensenanza/biblioteca_ele/publicaciones_centros/PDF/munich_20042005/06_crespo.pdf.

Brown, D. 2000. Principles of Language Learning and Teaching. (4ta. Ed.). Estados Unidos : s.n., 2000.

Caccuri, V. 2013. Educar con TICS. Buenos Aires : Fox Anidina, 2013.

Chacón Medina, A. (2003). La videoconferencia, conceptualización, elementos y uso educativo. Retrieved from http://www.ugr.es/ sevimeco/revistaeticanet/Numero2/Articulos/La\%20videoconferencia.pdf

Carucci, V. . 2015. Computación para docentes. . Buenos Aires : RedUsers, 2015.

Crystal, D. 2006. Language and the Internet . Cambridge : Cambridge, 2006.

Deans, P. C. 2009. Social Software and Web 2.0 Technology Trends. New York : Information Science Reference, 2009.

Domínguez C., G. 2012. El Rol del Docente Ante la Aplicación de Tecnologías para los Procesos Formativos. 2012. http://ilan.com.mx/wp-content/uploads/2012/10/RedILAN.pdf.

García, A. 2007. Principios de Interactividad: . s.l. : Televisión interactiva y realidad virtual. CES Felipe II, 3-10., 2007.

Gónzalez Vallés, J. E. 2015. Nuevas tendencias en innovación educativa superior. s.l. : ACCI, 2015.

Graells, P. 2005. La web 2.0 y sus aplicaciones didácticas. Departamento de pedagogía aplicada, Facultad de Educación UAB. s.l.: http://www.peremarques.net/web20.htm.

Gutiérrez Valderrama, M. (2011). Influencia de las Herramientas Pedagógicas en el Proceso de Enseñanza del inglés. Retrieved from http://www.funlam.edu.co/uploads/facultadeducacion/51_Influencia-herramientaspedagogicas.pdf 
Grupo Educare. (2011). Hardware y Software. Retrieved from https://computacioncpc.files.wordpress.com/2011/06/teorc3ada-hardware-y-software.pdf

Hernández, Requena, S. 2008. El modelo constructivista con las nuevas tecnologías: aplicado en el proceso de aprendizaje. s.l.: Retrieved from http://www.uoc.edu/rusc/5/2/dt/esp/hernandez.pdf, 2008.

Ilhch, J. (1972). De-schooling society. Harrow books.

ITILT. (2011). Tecnologías interactivas en la enseñanza de los idiomas. Retrieved from http://www.itilt.eu/sites/default/files/u3/itilt-manual/iTILT_Handout_SPANISH.pdf

Marqués, P. (2012). Libros digitales para mejor el aprendizaje. Retrieved from http://www.uab.cat/PDF/PDF_1341296217791_es.pdf

Martínez Gimeno, A., \& Torres Barzabal, L. (2013). Los entornos personales de aprendizaje (PLE). Del cómo enseñar al cómo aprender. Retrieved from file:///C:/Users/Usuario/Downloads/245-257-1-PB.pdf

Minguell, M. E. 2002. Interactividad e interacción. s.1.: Retrieved from file://C:/Users/Usuario/Downloads/Dialnet-InteractividadEInteraccion-1252603.pdf, 2002

Mohammed Abdul, J. F. 2009 . Herramientas Web 2.0 para el Aprendizaje Colaborativo. . s.1. : Retrieved from http://remo.det.uvigo.es/solite/attachments/038_Web\%202.0.pdf, 2009 .

Moirano, C. 2005. Internet Intranet Extranet Redes Privadas Virtuales (Túneles). s.1. : http://www.ccee.edu.uy/ensenian/catcomp/material/Internet8.pdf, 2005.

Niño, J. 2010. Aplicaciones Web 2.0 (Aplicaciones web). . s.l. : Editex., 2010.

Palomar Sánchez, M. J. 2009. Innovación y Experiencias Educativa. . s.1. : Retrieved from Ventajas e inconvenientes de las TICS en el aula: http://www.csicsif.es/andalucia/modules/mod_ense/revista/pdf/Numero_25/MARIA_JOSE_PALOMAR_S ANCHEZ01.pdf, 2009.

Pérez Vera, C. 2009. La Web 2.0 como recurso para la enseñanzael francés como lengua extranjera. Madrid : Ministerio de Educación, Cultura y Deporte., 2009.

Sarmiento Santana, M. 2007. Medios, Recursos, Materiales Multimedia. . s.1. : Retrieved from http://www.tdx.cat/bitstream/handle/10803/8927/FTESIS_CAPITULO_4.pdf?sequence=6, 2007.

Savin-Baden, M. (2010). A practical Guide To Using Second Life in Higher Education. Berkshire: McGraw-Hill.

Sharma, P., \& Barrett, B. (2010). Blended Learning: Using Technology in and beyond the classroom.Retrieved from https://www.researchgate.net/publication/220374249_Blended_Learning_Using_Technology _in_and_beyond_the_Language_Classroom

Shelly, G. B., \& Frydenberg, M. 2010. Web 2.0: Concepts and Applications. . Boston. : s.n., 2010. 
Thanky, P. (2014). The importance of English and Communication Skills for Technical Professionals. pg. 1.

Valerio, G. (2001). Transferencia. Retrieved from Revista Digital de Postgrado, Investigación y Extensión del Campus Monterrey: www.mty.itesm.mx/die/ddre/transferencia/57/57III.01.html 


\section{Para citar el artículo indexado.}

Herrera Andrade, Z., Colcha Guashpa, E., \& Barragán Murillo, R. de los Ángeles. (2019). La web 2.0 como herramienta interactiva para mejorar la comprensión lectora de textos en inglés para estudiantes universitarios. Explorador

\section{¿Ciencia}

El artículo que se publica es de exclusiva responsabilidad de los autores y no necesariamente reflejan el pensamiento de la Revista Explorador Digital.

El articulo queda en propiedad de la revista y, por tanto, su publicación parcial y/o total en otro medio tiene que ser autorizado por el director o editor de la Revista Explorador Digital.
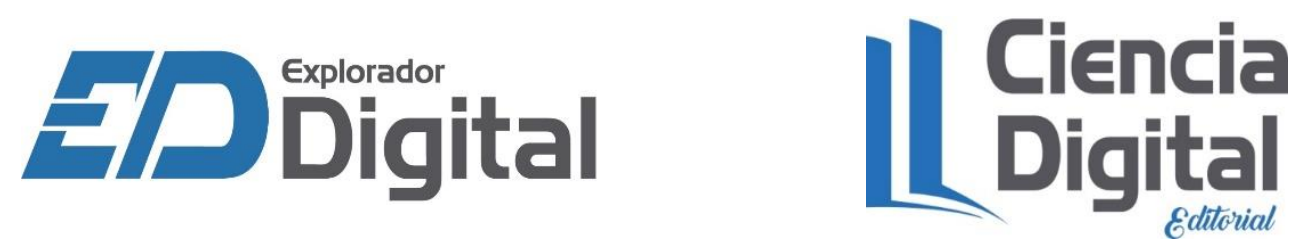